\title{
Time Trend of the Sensitivity of the Pneumococcal Urinary Antigen Test for Diagnosing Pneumococcal Community-Acquired Pneumonia: An Analysis of 15- Year, Prospective Cohort Data
}

\author{
Akihiro Ito (D) - Tadashi Ishida $\cdot$ Hiromasa Tachibana \\ Yosuke Nakanishi · Akio Yamazaki · Yasuyoshi Washio
}

Received: April 28, 2021 / Accepted: July 20, 2021 / Published online: August 2, 2021

(C) The Author(s) 2021

\section{ABSTRACT}

Introduction: Whether the sensitivity of the BinaxNOW Streptococcus pneumoniae urinary antigen test kit (BinaxNOW), adjusted by some variables including vital signs, laboratory examinations and pneumonia severity, has

\author{
A. Ito $(\bowtie) \cdot T$. Ishida $\cdot$ H. Tachibana \\ Y. Nakanishi · A. Yamazaki · Y. Washio \\ Department of Respiratory Medicine, Ohara \\ Healthcare Foundation, Kurashiki Central Hospital, \\ 1-1-1 Miwa, Kurashiki, Okayama 710-8602, Japan \\ e-mail: ai12306@kchnet.or.jp \\ T. Ishida \\ e-mail: ishidat@kchnet.or.jp \\ Y. Nakanishi \\ e-mail: yn16140@kchnet.or.jp \\ H. Tachibana \\ Department of Respiratory Medicine, National \\ Hospital Organization Minami Kyoto Hospital, 11 \\ Nakaashihara, Joyo, Kyoto, Japan \\ e-mail: tcbnh9@gmail.com \\ A. Yamazaki \\ Department of Respiratory Medicine, Shiga \\ University of Medical Science, Tsukinowa Seta-Cho, \\ Otsu, Shiga, Japan \\ e-mail: akio0420.1@gmail.com

\section{Y. Washio} \\ Graduate School of Medical Sciences, Research \\ Institute for Diseases of the Chest, Kyushu \\ University, 3-1-1 Higashiku, Maidashi, Fukuoka, \\ Japan \\ e-mail: yasuyoshi.washio@gmail.com
}

been decreasing is unknown. The aim of the present study was to investigate whether BinaxNOW sensitivity has decreased recently and to identify the predictors of the BinaxNOW result, including the time trend.

Methods: This prospective cohort study enrolled consecutive patients with pneumococcal community-acquired pneumonia who were hospitalised at Kurashiki Central Hospital from January 2001 to December 2015. Pneumococcal community-acquired pneumonia was defined as positive blood or pleural effusion or sputum culture results. To evaluate the effect of the time trend for the sensitivity of BinaxNOW, time series regression analysis was performed. In addition, predictors of the BinaxNOW result were examined by multivariable analysis using variables such as sex, vital signs, blood tests such as C-reactive protein, albumin, blood urea nitrogen, creatinine, white blood cell count, haematocrit and platelets, antibiotic pre-treatment, bacteraemia, and pneumonia severity, in addition to time trend and seasonality.

Results: A total of 446 patients were included. BinaxNOW sensitivity showed a significant, gradual decrease from $2001(81.3 \%)$ to 2015 $(48.7 \%)$. On multivariable analysis [odds ratio (95\% confidence interval)], bacteraemia [2.516 (1.387-4.561), $P=0.002$ ] was a predictor of a positive BinaxNOW result, whereas male sex [0.467 (0.296-0.736), $P=0.001]$, white blood cell count [0.959 (0.930-0.989), $P=0.008]$ and the time trend per year [0.900 (0.859-0.943), 
$P<0.001]$ were predictors of a negative BinaxNOW result.

Conclusions: The sensitivity of BinaxNOW decreased over a 15 -year period. We should be careful when interpreting BinaxNOW results in daily clinical practice, and the development of a new kit with good sensitivity is anticipated.

Trial registration number: UMIN000004353.

Keywords: Community-acquired pneumonia; Diagnosis; Pneumococcal pneumonia; Sensitivity; Urinary antigen test

\section{Key Summary Points}

\section{Why carry out this study?}

Some studies have reported that pneumonia severity and some variables including symptoms, vital signs and laboratory findings affect the sensitivity of the pneumococcal urinary antigen test.

Whether the sensitivity of the pneumococcal urinary antigen test, adjusted by some variables including vital signs, laboratory examinations and pneumonia severity, has truly decreased gradually over time is unknown.

The aim of the present study was to investigate whether BinaxNOW sensitivity has decreased recently and to identify the predictors of the BinaxNOW result, including the time trend.

\section{What was learned from the study?}

The time trend, male sex, low white blood cell counts and absence of bacteraemia were predictors of a negative BinaxNOW result.

We should use and interpret the BinaxNOW to diagnose pneumococcal community-acquired pneumonia on the basis of such background information, and the development of a new kit with good sensitivity is anticipated.

\section{INTRODUCTION}

Community-acquired pneumonia (CAP) is a leading cause of morbidity and mortality worldwide [1]. The cornerstone of CAP therapy is antibiotic agents, and assessment of the causative pathogens of pneumonia is important for selection of appropriate antibiotics. The most common causative microorganism is Streptococcus pneumoniae [2-5]. Culture of respiratory specimens is the gold standard for the diagnosis of pneumococcal pneumonia, but there are some weak points: it takes several days to identify the organism, and it is difficult to identify the organism with antibiotic pre-treatment [6].

Currently, a urinary antigen test (UAT) kit that detects the C-polysaccharide antigen of the cell wall of all types of S. pneumoniae for diagnosing pneumococcal pneumonia is widely used worldwide as a simple procedure with rapid results $[6,7]$. The specificity of the pneumococcal UAT was reported to be $90-100 \%$ [8-10], making it very useful for definite diagnosis. However, the sensitivity of the pneumococcal UAT for diagnosing pneumococcal pneumonia has been reported to be $65-100 \%$ $[8,9,11-18]$, with variation among studies. Recently, some reports showed that the sensitivity of the pneumococcal UAT was 50-60\% $[19,20]$. In addition, Shoji et al. [20] reported that the sensitivity of the pneumococcal UAT decreased significantly from 2001 (sensitivity in 2001-2005 was 76.4\%) to 2015 (sensitivity in 2011-2015 was 60.5\%). However, some studies have reported that pneumonia severity and some variables including symptoms, vital signs and laboratory findings affect the sensitivity of the pneumococcal UAT $[8,10]$, and Shoji et al.'s study did not adjust for these factors. Therefore, whether the sensitivity of the pneumococcal UAT has truly decreased gradually over time is unclear.

In Japan, there were some studies that investigated the sensitivity of the pneumococcal UAT $[9,21-24]$, but there are few studies that evaluated the time trend of the sensitivity of the pneumococcal UAT. Regarding the appropriate use and evaluation of the results of the 
pneumococcal UAT for diagnosing pneumococcal pneumonia, it is important to investigate and understand the time trend of the sensitivity of the pneumococcal UAT.

The aim of the present study was to investigate the time trend of the sensitivity of the pneumococcal UAT over a 15 -year period in Japan. The predictors of the pneumococcal UAT result were also examined to analyse whether the time trend significantly affected the sensitivity of the pneumococcal UAT.

\section{METHODS}

\section{Study Design and Setting}

Consecutive patients hospitalised with CAP at the Kurashiki Central Hospital, which is a 1166-bed tertiary hospital, were prospectively enrolled to evaluate clinical characteristics, causative pathogens, antibiotic regimens, and outcomes from July 1994. This prospective cohort study was registered with UMIN (UMIN000004353) in October 2010 and continues currently. The present study included the patients with pneumococcal CAP from January 2001 to December 2015 in the analysis from this prospective cohort data. This period was selected for two reasons. First, the results of the pneumococcal UAT were investigated in our prospective cohort study from September 2000. Second, the BinaxNOW S. pneumoniae UAT kit (Abbott Diagnostics Medical, Lake Forest, CA, USA) (BinaxNOW) was used as the UAT from September 2000 to June 2016. The patients were diagnosed with CAP if they had at least one of the clinical symptoms of fever, cough, sputum, dyspnoea, and pleuritic chest pain, plus at least one finding of coarse crackles on auscultation or elevated inflammatory biomarkers including C-reactive protein or white blood cell count, in addition to new infiltration shadows on chest radiography. The exclusion criteria were age $<15$ years, etiology of other than S. pneumoniae and unknown etiology, healthcare-associated pneumonia [25] and hospital-acquired pneumonia. Healthcare-associated pneumonia (HCAP) was diagnosed in patients who met at least one of the following criteria: (a) hospitalization for at least 2 days in the preceding 90 days; (b) residence in a nursing home or long-term care facility; (c) outpatients who needed intravenous therapy (including antibiotic agents and anticancer chemotherapy); (d) outpatients who needed dialysis (including haemodialysis and peritoneal dialysis); or (e) home wound care [25]. This study was performed as part of a clinical study of pneumonia (UMINO00004353) and was approved by the institutional review board of Kurashiki Central Hospital (approval number 3560). The study was performed in accordance with the ethical standards established in the 1964 Declaration of Helsinki and its later amendments. All patients gave their informed consent to participate in this study.

In all patients, characteristics including age, sex, comorbidities, preceding antimicrobial treatment before admission, history of pneumococcal vaccination within 5 years, vital signs on admission such as systolic blood pressure, blood examinations such as C-reactive protein, albumin, blood urea nitrogen, creatinine, white blood cell count, haematocrit, and platelet count on admission, causative pathogens, antibiotic regimens and 30-day mortality were investigated. The pneumonia severity on admission was evaluated using the CURB-65 score [confusion, urea $>7 \mathrm{mmol} / \mathrm{L}$, respiratory rate $\geq 30$ breaths per minute, low blood pressure (systolic $<90 \mathrm{mmHg}$ or diastolic $\leq 60 \mathrm{mmHg}$ ), and age $\geq 65$ years] [26] and the Pneumonia Severity Index (PSI) [27]. Both CURB-65 and PSI have been commonly used worldwide to assess pneumonia severity, site of care, and prognosis. In a systematic review and meta-analysis, overall performance of CURB-65 and PSI was similar for predicting mortality in CAP [28]. CURB-65 has five items including age, vital signs and laboratory examinations, and PSI has 20 items including age, sex, comorbidities, vital signs and laboratory examinations as variables.

\section{Criteria for Pneumococcal Pneumonia}

Sputum and blood for cultures and blood for measuring serum antibodies were collected on 
admission to detect causative pathogens. In addition, if the patients had a pleural effusion, the pleural effusion was collected to culture as much as possible. A causative pathogen was identified in accordance with the criteria in our previous report [29].

Patients were defined as having pneumococcal pneumonia if at least one of the following items was satisfied: (1) positive blood culture; (2) positive pleural fluid culture; or (3) positive sputum culture of greater than $1+$ on a qualitative test or $10^{5}$ on a quantitative test, referring to a significant Gram stain using only good quality sputum. A patient with only a positive BinaxNOW result was not considered to have pneumococcal pneumonia. Therefore, patients diagnosed with only a positive result of the pneumococcal UAT and without the pneumococcal UAT test were excluded from the analysis.

\section{Urinary Antigen Test for Diagnosing Pneumococcal Pneumonia}

BinaxNOW was used in our hospital from September 2000 to June 2016. The test was performed in accordance with the instructions of the manufacturer within $48 \mathrm{~h}$ after admission. The sensitivity of BinaxNOW was calculated as the number of positive results of the pneumococcal UAT/the number of pneumococcal CAP cases $\times 100$.

\section{Outcome}

The primary outcome of the present study was the time trend of the sensitivity of the pneumococcal UAT for diagnosing pneumococcal CAP from January 2001 to December 2015. Time trend means the change rates of the sensitivity of the pneumococcal UAT per year. The variables including age, sex, comorbidities, preceding antimicrobial treatment before admission, history of pneumococcal vaccination, vital signs, blood examinations and pneumonia severity that affect the sensitivity of BinaxNOW were investigated to determine whether the sensitivity was affected by a time trend.

\section{Statistical Analysis}

Categorical variables are expressed as numbers and percentage, whereas continuous variables are expressed as medians and interquartile range. Categorical variables were analysed using Fisher's exact test, and continuous variables were analysed using the non-parametric Mann-Whitney $U$ test.

To evaluate the effect of the time trend on BinaxNOW sensitivity, time series regression analysis was performed and adjusted by covariates that were significant on multivariable analysis in addition to seasonality.

To assess the predictors of a positive BinaxNOW result in patients with pneumococcal $\mathrm{CAP}$, univariate logistic regression analyses were performed for each variable. Then, a multivariable logistic regression analysis with stepwise backward elimination was performed using all variables with $P<0.05$ and covariates such as sex, systolic blood pressure, heart rate, blood urea nitrogen, antibiotic pre-treatment, bacteraemia, and CURB-65 based on previous reports $[8,10,20,30,31]$ and forced entry of the time trend and seasonality.

The seasonality of the sensitivity of BinaxNOW was assessed by considering two annual humps, which were accommodated by including four sine-cosine terms. Consecutive independence over time in the residuals was evaluated by autocorrelation plots.

All tests were two-tailed, and $P<0.05$ was considered significant. All statistical analyses were performed using $\mathrm{R}$ (version 3.0.3, Vienna, Austria).

\section{RESULTS}

\section{Patients' Characteristics}

The study flow chart is shown in Fig. 1. Of the total of 446 Japanese patients with pneumococcal CAP included, 274 (61.4\%) had a positive BinaxNOW result. The patients' clinical characteristics are listed in Table 1. Male sex was significantly associated with a negative BinaxNOW result. Age, comorbidities excluding chronic kidney diseases, preceding 


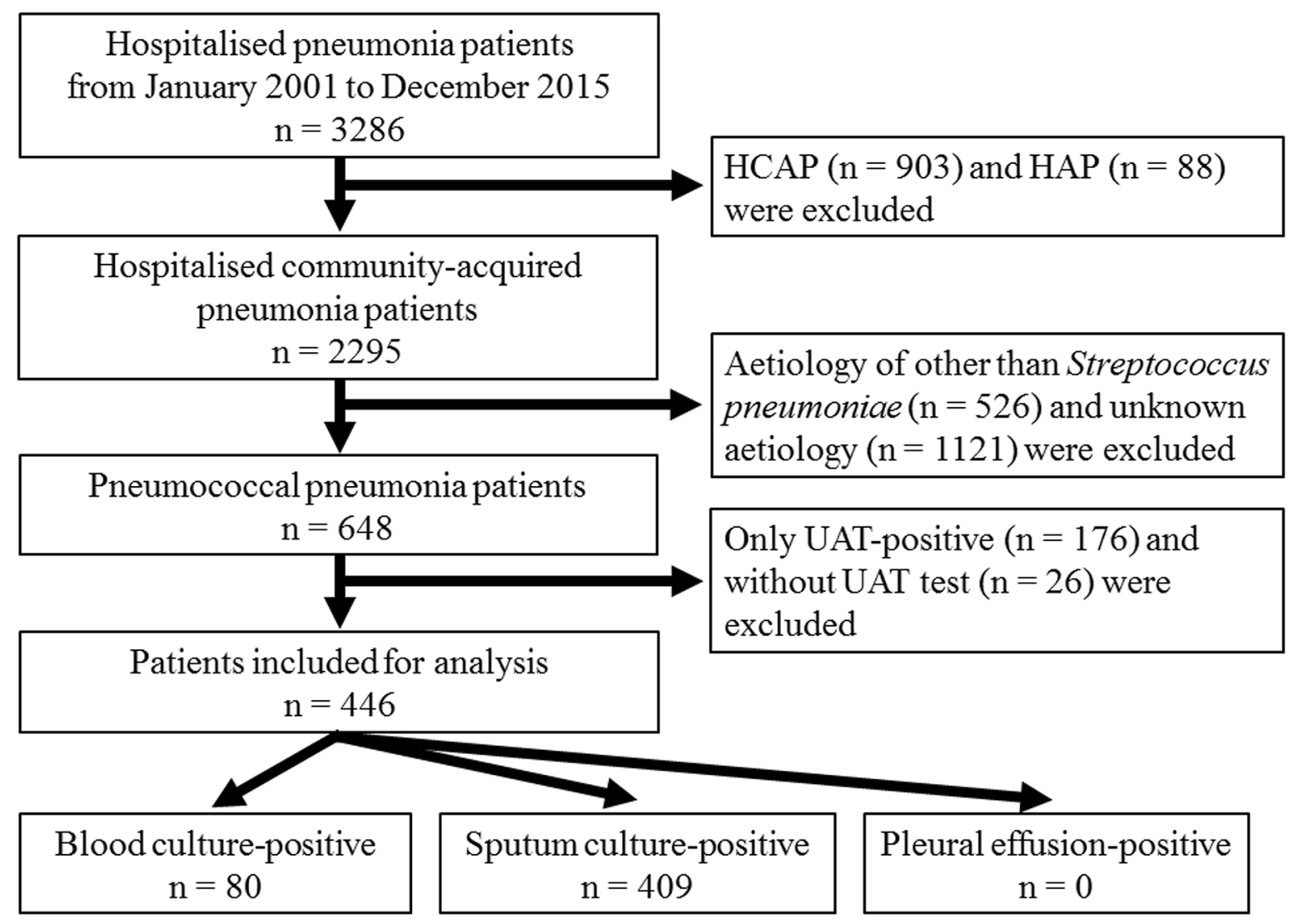

Fig. 1 Study flow chart

antimicrobial treatment, history of pneumococcal vaccination, all vital signs, blood laboratory examinations excluding C-reactive protein, albumin, white blood cell count and haematocrit, pneumonia severity scores and 30-day mortality were not significantly different between patients with positive and those with negative BinaxNOW results. Patients with bacteraemia were significantly more likely to have positive results.

\section{Changes in Sensitivity of Pneumococcal Urinary Antigen Test Between 2001 and 2015}

The sensitivity of BinaxNOW in the second half period (July 2007-December 2015) was significantly lower than in the first half period (January 2001-June 2007$) \quad(52.0 \%$ vs $71.0 \%$,
$P<0.001)$. In addition, the sensitivity of BinaxNOW in the last 2 years (January 2014-December 2015) was significantly lower than in the first 2 years (January 2001-December 2002$)(49.4 \%$ vs $79.3 \%, P<0.001)$.

Figure 2 shows BinaxNOW sensitivity and the rate of bacteraemia in each year between 2001 and 2015. BinaxNOW sensitivity decreased gradually from 2001 (81.3\%) to 2015 $(48.7 \%)$. The rate of bacteraemia ranged from $0 \%$ to $42.9 \%$ in $2001-2015$, although it varied in each year. Figure 2 also shows the curved lines of the predicted sensitivity of BinaxNOW and the 95\% confidence interval adjusted by sex, white blood cell count, bacteraemia and seasonality. The sensitivity of BinaxNOW in each year was within or near the predicted lines, with a significant negative slope.

Figure 3 shows the result of the autocorrelation function. The autocorrelation function was 
Table 1 Characteristics of patients with pneumococcal community-acquired pneumonia in those with positive and those with negative urinary antigen test results

\begin{tabular}{|c|c|c|c|c|}
\hline & $\begin{array}{l}\text { All patients } \\
n=446\end{array}$ & $\begin{array}{l}\text { Positive } \\
n=274\end{array}$ & $\begin{array}{l}\text { Negative } \\
n=172\end{array}$ & $P$ value \\
\hline Age (years) & $73[64-81]$ & $73[64-81]$ & $74[66-81]$ & 0.59 \\
\hline Male & $299(67.0)$ & $171(62.4)$ & $128(74.4)$ & 0.01 \\
\hline \multicolumn{5}{|l|}{ Comorbidity } \\
\hline COPD & $100(22.4)$ & $53(19.3)$ & $47(27.3)$ & 0.06 \\
\hline Chronic heart disease & $86(19.3)$ & $48(17.5)$ & $38(22.1)$ & 0.27 \\
\hline Diabetes mellitus & $67(15.0)$ & $36(13.1)$ & $31(18.0)$ & 0.17 \\
\hline Cerebrovascular disease & $42(9.4)$ & $25(9.1)$ & $17(9.9)$ & 0.87 \\
\hline Malignant disease $^{\mathrm{a}}$ & $26(5.8)$ & $18(6.6)$ & $8(4.7)$ & 0.53 \\
\hline Chronic liver disease & $26(5.8)$ & $18(6.6)$ & $8(4.7)$ & 0.53 \\
\hline Chronic kidney disease & $17(3.8)$ & $6(2.2)$ & $11(6.4)$ & 0.04 \\
\hline Pre-treatment of antibiotics & $65(14.6)$ & $40(14.6)$ & $25(14.5)$ & 1.00 \\
\hline Pneumococcal vaccination & & & & 0.16 \\
\hline Yes & $24(5.4)$ & $11(4.0)$ & $13(7.6)$ & \\
\hline Male & $16(3.6)$ & $4(1.5)$ & $12(7.0)$ & \\
\hline No & $52(11.7)$ & $29(10.6)$ & $23(13.4)$ & \\
\hline Male & $35(7.8)$ & $16(5.8)$ & $19(11.0)$ & \\
\hline Unknown & $370(83.0)$ & $234(85.4)$ & $136(79.1)$ & \\
\hline Male & $248(55.6)$ & $151(55.1)$ & $97(56.4)$ & \\
\hline \multicolumn{5}{|l|}{ Vital signs } \\
\hline Systolic blood pressure $(\mathrm{mmHg})$ & $125[108-141]$ & $124[108-140]$ & $125[109-142]$ & 1.00 \\
\hline Heart rate (beats/min) & $100[90-112]$ & $100[90-113]$ & $100[90-110]$ & 0.34 \\
\hline \multicolumn{5}{|l|}{ Laboratory findings } \\
\hline C-reactive protein $(\mathrm{mg} / \mathrm{L})$ & $162[79-246]$ & $173[99-257]$ & $137[60-208]$ & 0.001 \\
\hline Albumin $(\mathrm{g} / \mathrm{L})$ & $3.3[2.9-3.8]$ & $3.3[2.8-3.7]$ & $3.4[3.0-3.8]$ & 0.004 \\
\hline Blood urea nitrogen $(\mathrm{mg} / \mathrm{dL})$ & $19[13-27]$ & $19[13-28]$ & 19 [14-27] & 0.42 \\
\hline Creatinine $(\mathrm{mg} / \mathrm{dL})$ & $0.86[0.70-1.12]$ & $0.80[0.66-1.13]$ & $0.90[0.70-1.10]$ & 0.16 \\
\hline White blood cell count $\left(\times 10^{3} / \mu \mathrm{L}\right)$ & $11.6[8.2-16.0]$ & $11.0[8.0-15.5]$ & $12.4[9.1-17.1]$ & 0.01 \\
\hline Haematocrit (\%) & $37.5[33.9-40.7]$ & $36.7[33.5-40.4]$ & $38.0[35.5-41.2]$ & 0.004 \\
\hline Platelet count $\left(\times 10^{4} / \mu \mathrm{L}\right)$ & $18.6[14.1-24.2]$ & $18.9[14.4-25.0]$ & $18.4[13.7-23.1]$ & 0.43 \\
\hline Bacteraemia & $80(17.9)$ & $62(22.6)$ & $18(10.5)$ & 0.001 \\
\hline CURB-65 (score) & & & & 0.62 \\
\hline
\end{tabular}


Table 1 continued

\begin{tabular}{lllll}
\hline & $\begin{array}{l}\text { All patients } \\
\boldsymbol{n}=\mathbf{4 4 6}\end{array}$ & $\begin{array}{l}\text { Positive } \\
\boldsymbol{n}=\mathbf{2 7 4}\end{array}$ & $\begin{array}{l}\text { Negative } \\
\boldsymbol{n}=\mathbf{1 7 2}\end{array}$ & P value \\
\hline 0 & $53(11.9)$ & $28(10.2)$ & $25(14.5)$ & \\
1 & $123(27.6)$ & $79(28.8)$ & $44(25.6)$ & \\
2 & $143(32.1)$ & $86(31.4)$ & $31(33.1)$ & \\
3 & $86(19.3)$ & $55(20.1)$ & $14(8.1)$ & \\
4 & $33(7.4)$ & $19(6.9)$ & $1(0.6)$ & 0.59 \\
5 & $6(1.3)$ & $5(1.8)$ & $0(0)$ & \\
Unknown & $2(0.4)$ & $2(0.7)$ & $5(2.9)$ & \\
PSI (class) & & & $26(15.1)$ \\
I & $17(3.8)$ & $12(4.4)$ & $54(31.4)$ & \\
II & $68(15.2)$ & $42(15.3)$ & $71(41.3)$ & \\
III & $129(28.9)$ & $75(27.4)$ & $16(9.3)$ & \\
IV & $177(39.7)$ & $106(38.7)$ & $0(0)$ & \\
V & $53(11.9)$ & $37(13.5)$ & $6(3.5)$ & \\
Unknown & $2(0.4)$ & $2(0.7)$ & $10(3.6)$ & \\
30 -day mortality & $16(3.6)$ & &
\end{tabular}

Data are presented as medians and interquartile range or $n(\%)$

$C O P D$ chronic obstructive pulmonary disease, $C U R B-65$ confusion, urea $>7 \mathrm{mmol} / \mathrm{L}$, respiratory rate $\geq 30$ breaths $/ \mathrm{min}$, low blood pressure (systolic $<90 \mathrm{mmHg}$ or diastolic $\leq 60 \mathrm{mmHg}$ ), and age $\geq 65$ years, PSI Pneumonia Severity Index

a Those that were active on admission or were diagnosed within 1 year of admission were included

generally within $95 \%$ confidence intervals (blue dash lines); therefore, the effect of autocorrelation was considered to be minimal.

\section{Predictors of Pneumococcal Urinary Antigen Test Result}

On multivariable analysis [odds ratio (95\% confidence interval)], bacteraemia [2.516 (1.387-4.561), $P=0.002]$ was a predictor of a positive BinaxNOW result, whereas male sex [0.467 (0.296-0.736), $P=0.001]$, white blood cell count [0.959 (0.930-0.989), $P=0.008]$ and the time trend per year [0.900 (0.859-0.943), $P<0.001]$ were predictors of a negative BinaxNOW result (Table 2 ).

\section{DISCUSSION}

The present study demonstrated that BinaxNOW sensitivity showed a significant gradual decrease over a 15-year period after adjusting for covariates including sex, white blood cell count, bacteraemia and seasonality. The sensitivity of BinaxNOW in 2001 was $81.3 \%$, but it was less than $50 \%$ in 2015. Furthermore, on multivariable analysis, the time trend per year was a predictor of a negative BinaxNOW result, in addition to male sex, high white blood cell count and absence of bacteraemia.

Regarding the time trend of the sensitivity of BinaxNOW, there have been no reports of longterm investigations in Japan, although some previous studies $[9,21-24]$ reported BinaxNOW 


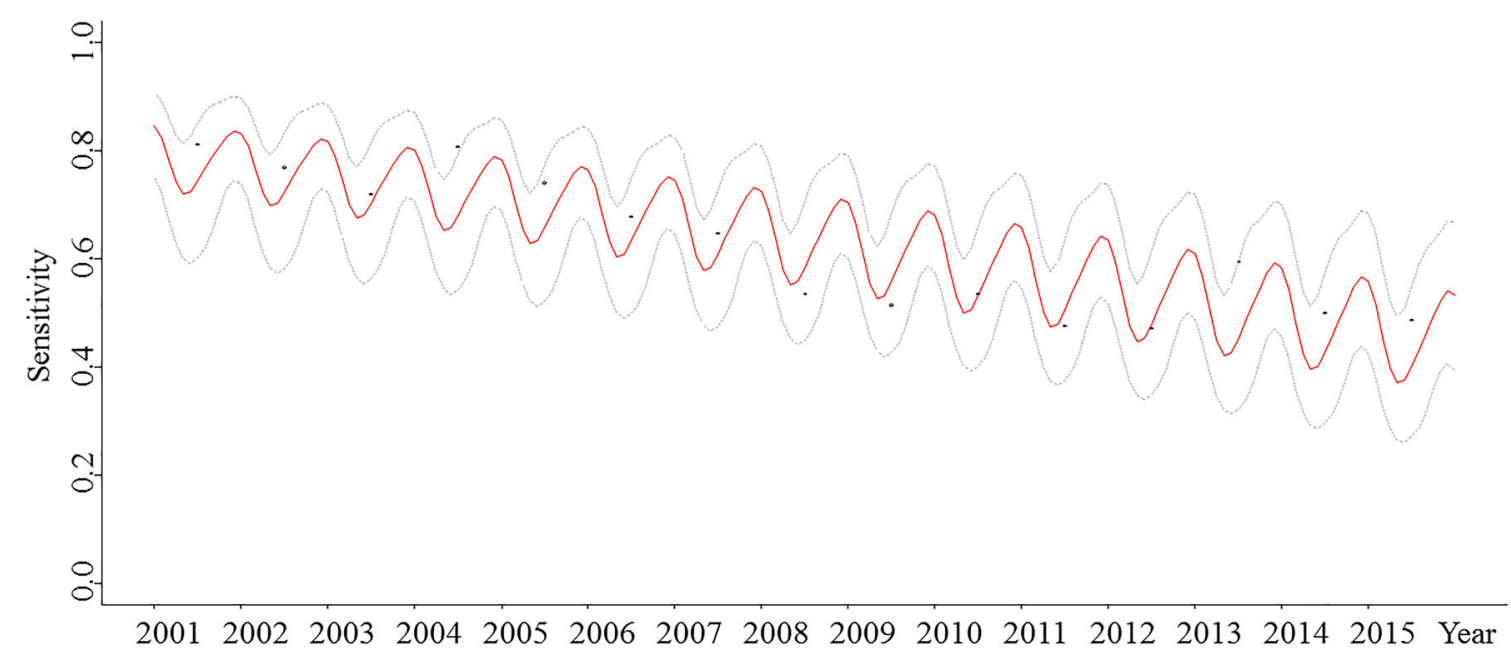

\begin{tabular}{|c|c|c|c|c|c|c|c|c|c|c|c|c|c|c|c|}
\hline Year & 2001 & 2002 & 2003 & 2004 & 2005 & 2006 & 2007 & 2008 & 2009 & 2010 & 2011 & 2012 & 2013 & 2014 & 2015 \\
\hline $\begin{array}{c}\text { Sensitivity, } \\
\%\end{array}$ & $\begin{array}{c}81.3 \\
(26 / 32)\end{array}$ & $\begin{array}{c}76.9 \\
(20 / 26)\end{array}$ & $\begin{array}{c}72.0 \\
(18 / 25)\end{array}$ & $\begin{array}{c}80.8 \\
(21 / 26)\end{array}$ & $\begin{array}{c}74.1 \\
(20 / 27)\end{array}$ & $\begin{array}{c}67.9 \\
(19 / 28)\end{array}$ & $\begin{array}{c}64.7 \\
(22 / 34)\end{array}$ & $\begin{array}{c}53.6 \\
(15 / 28)\end{array}$ & $\begin{array}{c}51.4 \\
(18 / 35)\end{array}$ & $\begin{array}{c}53.6 \\
(15 / 28)\end{array}$ & $\begin{array}{c}47.6 \\
(10 / 21)\end{array}$ & $\begin{array}{c}47.1 \\
(8 / 17)\end{array}$ & $\begin{array}{c}59.4 \\
(19 / 32)\end{array}$ & $\begin{array}{c}50.0 \\
(24 / 48)\end{array}$ & $\begin{array}{c}48.7 \\
(19 / 39)\end{array}$ \\
\hline $\begin{array}{c}\text { Bacteraemia, } \\
\%\end{array}$ & $\begin{array}{c}12.5 \\
(4 / 32)\end{array}$ & $\begin{array}{c}19.2 \\
(5 / 26)\end{array}$ & $\begin{array}{c}16.0 \\
(4 / 25)\end{array}$ & $\begin{array}{c}26.9 \\
(7 / 26)\end{array}$ & $\begin{array}{c}3.7 \\
(1 / 27)\end{array}$ & $\begin{array}{c}32.1 \\
(9 / 28)\end{array}$ & $\begin{array}{c}23.5 \\
(8 / 34)\end{array}$ & $\begin{array}{c}42.9 \\
(12 / 28)\end{array}$ & $\begin{array}{c}11.4 \\
(4 / 35)\end{array}$ & $\begin{array}{c}21.4 \\
(6 / 28)\end{array}$ & $\begin{array}{c}14.3 \\
(3 / 21)\end{array}$ & $\begin{array}{c}0 \\
(0 / 17)\end{array}$ & $\begin{array}{c}12.5 \\
(4 / 32)\end{array}$ & $\begin{array}{c}14.6 \\
(7 / 48)\end{array}$ & $\begin{array}{c}15.4 \\
(6 / 39)\end{array}$ \\
\hline
\end{tabular}

Fig. 2 Sensitivity of the pneumococcal urinary antigen test in annual data and predictions of the harmonic model. The dots show the sensitivity of the pneumococcal urinary antigen test each year from 2001 to 2015 . The red curved line shows the predicted sensitivity of BinaxNOW, and the grey lines show the $95 \%$ confidence interval. They were adjusted by sex, white blood cell count, bacteraemia and

sensitivity over the short term, such as 1-3 years. Therefore, the present study was the first that evaluated the time trend of the sensitivity of BinaxNOW for diagnosing pneumococcal CAP for a long period of 15 years. Ishida et al. [9] reported that the sensitivity of BinaxNOW was $75.9 \%$ in patients with pneumococcal CAP in 2004. After that, some studies showed that BinaxNOW sensitivity in pneumococcal CAP was in the range of 53.7-62.0\% [21-24], which was lower compared with Ishida et al.'s study. The present study also showed that BinaxNOW sensitivity in the last 2 years (2014-2015) was relatively low (49.4\%), which implies that a negative BinaxNOW result could not rule out the diagnosis of pneumococcal CAP. Therefore, a new pneumococcal UAT kit with improved sensitivity is needed, although BinaxNOW has high specificity [8-10].

Concerning the reasons why BinaxNOW sensitivity has been decreasing, Shoji et al. [20] seasonality. Sensitivity of BinaxNOW and the rate of bacteraemia are shown in the table under the graph of the sensitivity of BinaxNOW. The number of positive results of BinaxNOW, bacteraemia, and patients with pneumococcal community-acquired in each year are also shown. BinaxNOW, BinaxNOW S. pneumoniae urinary antigen test kit

reported the possibility of S.pneumoniae serotype changes. They showed that the sensitivity of BinaxNOW was $76.4 \%, 77.9 \%$, and $60.5 \%$ in the periods from 2001 to 2005, from 2006 to 2010 and from 2011 to 2015, respectively, in Spain. They also showed the distribution of $S$. pneumoniae serotypes and the sensitivity of BinaxNOW for each serotype. The sensitivity of BinaxNOW was significantly higher for each serotype included in pneumococcal conjugate vaccine 13 (PCV13) than for non-PCV13 serotypes. The non-PCV13 serotypes increased gradually over the three periods $(20.5 \%$ from 2001 to $2005,35.8 \%$ from 2006 to $2010,50.6 \%$ from 2011 to 2015); therefore, they concluded that the sensitivity of BinaxNOW would be decreased [20]. However, their study did not adjust the confounding factors such as patients' background characteristics, pneumonia severity, and seasonality. Consequently, the present study is significant in that it showed the time 


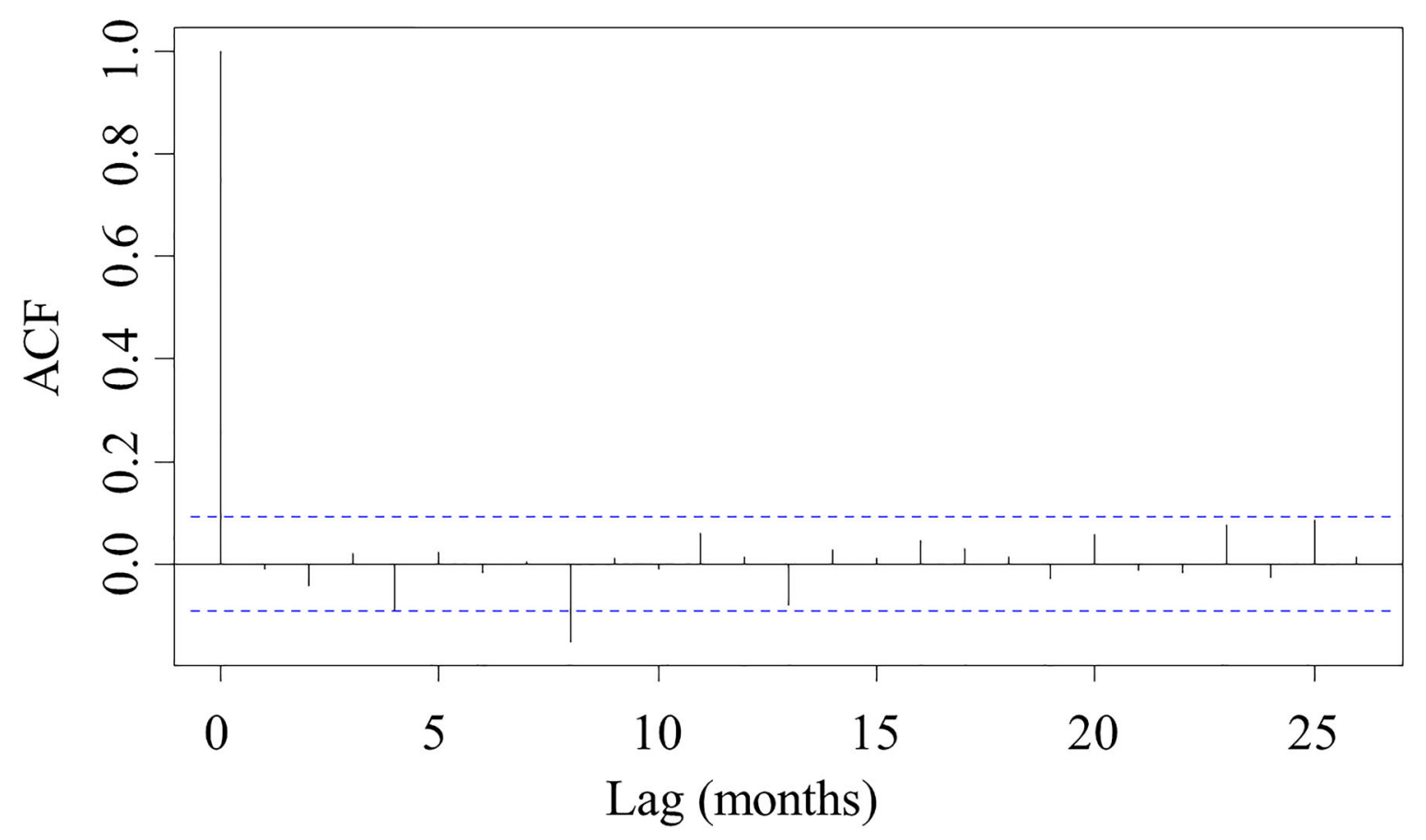

Fig. 3 Autocorrelation function $(\mathrm{ACF})$ in each time lag per month

trend and the decrease in BinaxNOW sensitivity by adjusting some covariates, including seasonality.

In Japan, pneumococcal polysaccharide vaccine (PPSV23) was introduced for adult patients at risk of pneumococcal disease for preventing pneumococcal infection from 1992. Furthermore, PCV7 vaccination was started for children in February 2010, and PCV7 was changed to PCV13 in November 2013. Regarding the serotype of adult invasive pneumococcal disease, Japanese surveillance showed that the distribution of non-PCV13 serotypes was $38.5 \%$ from 2006 to 2007 [32], and it increased to $54.0 \%$ in 2013 [33]. In addition, adult pneumococcal pneumonia cases caused by non-PCV13 serotypes increased from $47 \%$ in $2011-2014$ to $67 \%$ in 2016-2017 [34]. Therefore, in the present study, changes in the distribution of serotypes of $S$. pneumoniae may have affected the sensitivity of BinaxNOW, although the serotypes of S. pneumoniae were not identified.
On the other hand, Choi et al.'s study in Korea reported that the sensitivity of BinaxNOW was not decreased during the pre-PCV13 period in 2007-2009, the period immediately after paediatric PCV13 use in 2010-2011, and the post-PCV13 period in $2012-2013$ (53.4\% vs $51.1 \%$ vs $66.1 \%$, respectively) [19]. There are two possible reasons why the present study and Choi et al.'s study showed different results. First, the sensitivity of BinaxNOW in Choi et al.'s study was very low in 2007-2009 (53.4\%) compared with the present study. Second, the rate of bacteraemia in Choi et al.'s study was $4.4 \%$, lower than in the present study (17.9\%). In patients with pneumococcal pneumonia and bacteraemia, BinaxNOW usually has high sensitivity $[8,20,35]$, including in the present study. Therefore, the low rate of bacteraemia might have affected the sensitivity of BinaxNOW in Choi et al.'s study.

Regarding the predictors of the pneumococcal UAT in CAP, Molinos et al. showed that female sex, heart rate $\geq 125 / \mathrm{min}$, systolic blood 
Table 2 Predictors of a positive pneumococcal urinary antigen test

\begin{tabular}{|c|c|c|c|c|}
\hline & \multicolumn{2}{|c|}{ Univariate analysis (forced entry) } & \multicolumn{2}{|l|}{$\begin{array}{l}\text { Multivariable analysis (stepwise } \\
\text { backward elimination) }\end{array}$} \\
\hline & $\begin{array}{l}\text { Odds ratio ( } 95 \% \text { confidence } \\
\text { interval) }\end{array}$ & $P$ value & $\begin{array}{l}\text { Odds ratio (95\% confidence } \\
\text { interval) }\end{array}$ & $P$ value \\
\hline Male & $0.571[0.375-0.869]$ & 0.009 & $0.467[0.296-0.736]$ & 0.001 \\
\hline Chronic kidney disease & $0.328[0.119-0.903]$ & 0.03 & & \\
\hline Systolic blood pressure & $1.000[0.993-1.008]$ & 0.943 & & \\
\hline Heart rate & $1.007[0.996-1.018]$ & 0.20 & & \\
\hline C-reactive protein & $1.030[1.011-1.049]$ & 0.002 & & \\
\hline Albumin & $0.611[0.437-0.854]$ & 0.004 & & \\
\hline Blood urea nitrogen & $1.011[0.998-1.025]$ & 0.11 & & \\
\hline White blood cell count & $0.967[0.941-0.994]$ & 0.02 & $0.959[0.930-0.989]$ & 0.008 \\
\hline Haematocrit & $0.995[0.979-1.013]$ & 0.60 & & \\
\hline Pre-treatment of antibiotics & $1.005[0.585-1.726]$ & 0.99 & & \\
\hline Bacteraemia & $2.502[1.423-4.399]$ & 0.001 & $2.516[1.387-4.561]$ & 0.002 \\
\hline CURB-65 score & $1.067[0.905-1.259]$ & 0.439 & & \\
\hline Time trend ${ }^{\mathrm{a}}$ & $0.901[0.861-0.942]$ & $<0.001$ & $0.900[0.859-0.943]$ & $<0.001$ \\
\hline $\begin{array}{l}\text { Seasonal harmonic sin } \\
\qquad(\pi \times(\text { month/6) })\end{array}$ & $1.147[0.851-1.546]$ & 0.37 & $0.958[0.695-1.322]$ & 0.80 \\
\hline $\begin{array}{l}\text { Seasonal harmonic sin } \\
\qquad(\pi \times(\text { month } / 3))\end{array}$ & $1.017[0.774-1.335]$ & 0.90 & $1.061[0.795-1.416]$ & 0.69 \\
\hline $\begin{array}{l}\text { Seasonal harmonic cos } \\
\qquad(\pi \times(\text { month } / 6))\end{array}$ & $1.492[1.136-1.959]$ & 0.004 & $1.434[1.075-1.912]$ & 0.01 \\
\hline $\begin{array}{l}\text { Seasonal harmonic cos } \\
\qquad(\pi \times(\text { month } / 3))\end{array}$ & $1.040[0.778-1.391]$ & 0.79 & $1.012[0.745-1.375]$ & 0.94 \\
\hline
\end{tabular}

CURB-65 confusion, urea $>7 \mathrm{mmol} / \mathrm{L}$, respiratory rate $\geq 30$ breaths $/ \mathrm{min}$, low blood pressure (systolic $<90 \mathrm{mmHg}$ or diastolic $\leq 60 \mathrm{mmHg}$ ), and age $\geq 65$ years

a Time trend means the change rates of the sensitivity of the pneumococcal urinary antigen test per year

pressure $<90 \mathrm{mmHg}, \mathrm{SaO}_{2}<90 \%$, absence of prior antibiotic treatment, pleuritic chest pain, chills, pleural effusion, and blood urea nitrogen $\geq 30 \mathrm{mg} / \mathrm{dL}$ were significant predictors of a positive result [10]. The present study included all covariates excluding $\mathrm{SaO}_{2}<90 \%$, pleuritic chest pain and chills in the multivariable analysis, because these data were not collected prospectively in many patients. The results of the present study may have been different if these three items $\left(\mathrm{SaO}_{2}<90 \%\right.$, pleuritic chest pain and chills) had been included in the multivariable analysis. However, pneumonia severity scores such as CURB-65 [26] were included. This score includes evaluation of respiratory status, such as a respiratory rate $\geq 30$ breaths/ min. In addition, chills are reported to be a predictor of true bacteraemia [35-37]. Bacteraemia was included as one covariate, although Molinos et al.'s study did not include 
bacteraemia in the analysis. Therefore, the result of the present study appears reasonable.

The present study found that female sex, low white blood cell count and bacteraemia were predictors of a positive BinaxNOW result. As mentioned above, Molinos et al. [10] also reported that female sex was one of the predictors of a positive BinaxNOW result. The reason why the sensitivity of BinaxNOW was significantly higher in female patients than in male patients is unclear. However, it is possible that urinary antigen is excreted much more in women than in men, though there is no evidence to support this. Another possible reason is that many more male than female patients may have had pneumococcal vaccination as a result of chronic obstructive pulmonary disease (COPD). Concerning bacteraemia, some reports have shown that BinaxNOW had higher sensitivity in patients with bacteraemia than in those without $[8,20,30]$. In bacteraemic pneumococcal pneumonia, there is a larger bacterial load than in non-bacteraemic cases; therefore, pneumococcal capsule antigen is thought to be excreted in urine, which could lead to the BinaxNOW having high sensitivity [14, 38, 39].

In the present study, the sensitivity of BinaxNOW was about 50\% in 2010, and it was lower compared with the previous meta-analysis that examined the sensitivity of the pneumococcal UAT (pooled sensitivity 75\%, 95\% CI 71-79\%) [40]. Recently, some studies have reported that the sensitivity of BinaxNOW was $50-60 \%[19,20]$, similar to the present result. In our view, the sensitivity of the UAT should be much higher for effective use in daily clinical practice, although the specificity is very high (90-99\%) $[9,10,41]$. On the basis of the result of the present study, care is needed when interpreting the BinaxNOW result, even when the result is negative, especially in male patients or those without bacteraemia.

This study had some limitations. First, only hospitalised patients were included; therefore, these results could be overestimated because outpatients would have less severe disease or more would be non-bacteraemic. Second, this was a single-centre study, and whether the results of the present study could apply to other areas or other countries is unclear, because the rate of pneumococcal vaccination and the distribution of serotypes of $S$. pneumoniae differ in each area and country. Third, pneumococcal vaccine status could not be adjusted for because pneumococcal vaccine status was not investigated in most patients (83.0\%). Finally, the serotypes of S. pneumoniae were not identified; therefore, whether the change in the distribution of serotypes would affect the sensitivity of BinaxNOW is unknown. The strengths of the present study were that it was relatively large scale, including about 2300 patients with CAP, and it examined a long period of 15 years.

\section{CONCLUSIONS}

A significant decrease in the sensitivity of BinaxNOW for diagnosing pneumococcal CAP occurred gradually after adjusting for some covariates including sex, white blood cell count, bacteraemia and seasonality over a 15-year period in Japan. In addition to the time trend, male sex, low white blood cell count and absence of bacteraemia were predictors of a negative BinaxNOW result. We should use and interpret the BinaxNOW to diagnose pneumococcal CAP on the basis of such background information, and the development of a new kit with good sensitivity and specificity is anticipated.

\section{ACKNOWLEDGEMENTS}

We thank the participants of the study.

Funding. This research did not receive any specific grant from funding agencies in the public, commercial, or not-for-profit sectors. The journal's Rapid Service Fee was funded by the authors.

Authorship. All named authors meet the International Committee of Medical Journal Editors (ICMJE) criteria for authorship for this article, take responsibility for the integrity of the work as a whole, and have given their approval for this version to be published. 
Author Contributions. Akihiro Ito served as the principal author, had full access to all data in the study, and takes responsibility for the integrity and accuracy of the data and data analysis. Akihiro Ito, Tadashi Ishida, Hiromasa Tachibana, Yosuke Nakanishi, Akio Yamazaki and Yasuyoshi Washio contributed to the study conception and design and to the acquisition of data. Akihiro Ito, Tadashi Ishida, and Hiromasa Tachibana contributed to the analysis and interpretation of data. Akihiro Ito, Tadashi Ishida, Hiromasa Tachibana, Yosuke Nakanishi, Akio Yamazaki and Yasuyoshi Washio contributed to the drafting and revision of the manuscript and approved the final version to be submitted for consideration for publication.

Disclosures. Akihiro Ito, Tadashi Ishida, Hiromasa Tachibana, Yosuke Nakanishi, Akio Yamazaki and Yasuyoshi Washio have nothing to disclose.

Compliance with Ethics Guidelines. This study was performed as part of a clinical study of pneumonia (UMIN000004353) and was approved by the institutional review board of Kurashiki Central Hospital (approval number 3560). The study was performed in accordance with the ethical standards established in the 1964 Declaration of Helsinki and its later amendments. All patients gave their informed consent to participate in this study.

Data Availability. The datasets used and/or analysed during the current study are available from the corresponding author on reasonable request.

Open Access. This article is licensed under a Creative Commons Attribution-NonCommercial 4.0 International License, which permits any non-commercial use, sharing, adaptation, distribution and reproduction in any medium or format, as long as you give appropriate credit to the original author(s) and the source, provide a link to the Creative Commons licence, and indicate if changes were made. The images or other third party material in this article are included in the article's Creative Commons licence, unless indicated otherwise in a credit line to the material. If material is not included in the article's Creative Commons licence and your intended use is not permitted by statutory regulation or exceeds the permitted use, you will need to obtain permission directly from the copyright holder. To view a copy of this licence, visit http://creativecommons.org/licenses/by$\mathrm{nc} / 4.0 /$.

\section{REFERENCES}

1. Arnold FW, Wiemken TL, Peyrani P, Ramirez JA, Brock GN, for the CAPO authors. Mortality differences among hospitalized patients with community-acquired pneumonia in three world regions: results from the Community-Acquired Pneumonia Organization (CAPO) International Cohort Study. Respir Med. 2013;107:1101-11.

2. Ishida $\mathrm{T}$, Hashimoto $\mathrm{T}$, Arita $\mathrm{M}$, Ito I, Osawa M. Etiology of community-acquired pneumonia in hospitalized patients: a 3-year prospective study in Japan. Chest. 1998;114:1588-93.

3. Miyashita N, Fukano H, Niki Y, Matsushima T, Okimoto N. Etiology of community-acquired pneumonia requiring hospitalization in Japan. Chest. 2001;119:1295-6.

4. Mandell LA, Wunderink RG, Anzueto A, et al. Infectious Diseases Society of America/American Thoracic Society consensus guidelines on the management of community-acquired pneumonia in adults. Clin Infect Dis. 2007;44(Suppl. 2):S27-72.

5. Cillóniz C, Ewig S, Polverino E, et al. Microbial aetiology of community-acquired pneumonia and its relation to severity. Thorax. 2011;66:340-6.

6. Blaschke AJ. Interpreting assays for the detection of Streptococcus pneumoniae. Clin Infect Dis. 2011;52(Suppl 4):S331-7.

7. Werno AM, Murdoch DR. Medical microbiology: laboratory diagnosis of invasive pneumococcal disease. Clin Infect Dis. 2008;46:926-32.

8. Rosón B, Fernández-Sabé N, Carratalà J, et al. Contribution of a urinary antigen assay (Binax NOW) to the early diagnosis of pneumococcal pneumonia. Clin Infect Dis. 2004;38:222-6.

9. Ishida T, Hashimoto T, Arita M, Tojo Y, Tachibana $\mathrm{H}$, Jinnai M. A 3-year prospective study of a urinary antigen-detection test for Streptococcus pneumoniae in community-acquired pneumonia: utility and 
clinical impact on the reported etiology. J Infect Chemother. 2004;10:359-63.

10. Molinos L, Zalacain R, Menéndez R, et al. Sensitivity, specificity, and positivity predictors of the pneumococcal urinary antigen test in communityacquired pneumonia. Ann Am Thorac Soc. 2015;12: 1482-9.

11. Burel E, Dufour P, Gauduchon V, Jarraud S, Etienne J. Evaluation of a rapid immunochromatographic assay for detection of Streptococcus pneumoniae antigen in urine samples. Eur J Clin Microbiol Infect Dis. 2001;20:840-1.

12. Strålin K, Kaltoft MS, Konradsen HB, Olcén P, Holmberg H. Comparison of two urinary antigen tests for establishment of pneumococcal etiology of adult community-acquired pneumonia. J Clin Microbiol. 2004;42:3620-5.

13. Genné D, Siegrist HH, Lienhard R. Enhancing the etiologic diagnosis of community-acquired pneumonia in adults using the urinary antigen assay (Binax NOW). Int J Infect Dis. 2006;10:124-8.

14. Lasocki S, Scanvic A, Turdu FL, et al. Evaluation of the Binax NOW Streptococcus pneumoniae urinary antigen assay in intensive care patients hospitalized for pneumonia. Intensive Care Med. 2006;32: 1766-72.

15. Vidal J, Adrover A, García-Méndez E, García-Rey C, Dal-Ré R. Lack of usefulness of the immunochromatographic urinary pneumococcal antigen test in maximizing pneumococcal yield in a communityacquired pneumonia clinical trial. J Chemother. 2006;18:562-4.

16. del Mar G-S, Cima-Cabal MD, Villaverde R, et al. Performance of a pneumolysin enzyme-linked immunosorbent assay for diagnosis of pneumococcal infections. J Clin Microbiol. 2007;45: 3549-54.

17. Weatherall C, Paoloni R, Gottlieb T. Point-of-care urinary pneumococcal antigen test in the emergency department for community acquired pneumonia. Emerg Med J. 2008;25:144-8.

18. Sordé R, Falcó V, Lowak M, et al. Current and potential usefulness of pneumococcal urinary antigen detection in hospitalized patients with community-acquired pneumonia to guide antimicrobial therapy. Arch Intern Med. 2011;171:166-72.

19. Choi MJ, Song JY, Cheong HJ, et al. Clinical usefulness of pneumococcal urinary antigen test, stratified by disease severity and serotypes. J Infect Chemother. 2015;21:672-9.
20. Shoji H, Domenech A, Simonetti AF, et al. The Alere BinaxNOW pneumococcal urinary antigen test: diagnostic sensitivity for adult pneumococcal pneumonia and relationship to specific serotypes. J Clin Microbiol. 2018;56:e00787-e817.

21. Ehara N, Fukushima K, Kakeya H, et al. A novel method for rapid detection of Streptococcus pneumoniae antigen in sputum and its application in adult respiratory tract infections. J Med Microbiol. 2008;57:820-6.

22. Izumikawa K, Akamatsu S, Kageyama A, et al. Evaluation of a rapid immunochromatographic ODK0501 assay for detecting Streptococcus pneumoniae antigen in sputum samples from patients with lower respiratory tract infection. Clin Vaccine Immunol. 2009;16:672-8.

23. Fukushima K, Nakamura S, Inoue Y, et al. Utility of a sputum antigen detection test in pneumococcal pneumonia and lower respiratory infectious disease in adults. Intern Med. 2015;54:2843-50.

24. Ikegame S, Nakano T, Otsuka J, et al. The evaluation of the sputum antigen kit in the diagnosis of pneumococcal pneumonia. Intern Med. 2017;56: 1141-6.

25. American Thoracic Society; Infectious Diseases Society of America. Guidelines for the management of adults with hospital-acquired, ventilator-associated, and healthcare-associated pneumonia. Am J Respir Crit Care Med. 2005;171:388-416.

26. Lim WS, van der Eerden MM, Laing $\mathrm{R}$, et al. Defining community-acquired pneumonia severity on presentation to hospital: an international derivation and validation study. Thorax. 2003;58: 377-82.

27. Fine MJ, Auble TE, Yealy DM, et al. A prediction rule to identify low-risk patients with community-acquired pneumonia. N Engl J Med. 1997;336:243-50.

28. Chalmers JD, Singanayagam A, Akram AR, et al. Severity assessment tools for predicting mortality in hospitalised patients with community-acquired pneumonia. Systematic review and meta-analysis. Thorax. 2010;65:878-83.

29. Ito A, Ishida $\mathrm{T}$, Tokumasu $\mathrm{H}$, et al. Prognostic factors in hospitalized community-acquired pneumonia: a retrospective study of a prospective observational cohort. BMC Pulm Med. 2017;17:78. https://doi.org/10.1186/s12890-017-0424-4.

30. Murdoch DR, Laing RT, Mills GD, et al. Evaluation of a rapid immunochromatographic test for detection of Streptococcus pneumoniae antigen in urine samples from adults with community-acquired pneumonia. J Clin Microbiol. 2001;39:3495-8. 
31. Zhou F, Gu L, Qu JX, Liu YM, Cao B, for CAP-China network. Evaluating the utility of Binax NOW Streptococcus pneumoniae urinary antigen test in adults with community acquired pneumonia in China. Clin Respir J. 2018;12:425-32.

32. Chiba N, Morozumi M, Sunaoshi K, et al. Serotype and antibiotic resistance of isolates from patients with invasive pneumococcal disease in Japan. Epidemiol Infect. 2010;138:61-8.

33. Chang B, Amemura-Maekawa J. Bacteriological analysis of adult invasive pneumococcal diseasederived strains, Health and Labor Science Research Award subsidy, Emerging/Re-emerging Infectious Diseases Research Project 2014 General/Shared Research Report. 2015;2015:63-7 (in Japanese).

34. Sando E, Suzuki M, Furumoto A, et al. Impact of the pediatric 13-valent pneumococcal conjugate vaccine on serotype distribution and clinical characteristics of pneumococcal pneumonia in adults: the Japan Pneumococcal Vaccine Effectiveness Study (JPAVE). Vaccine. 2019;37:2687-93.

35. Tokuda Y, Miyasato H, Stein GH. A simple prediction algorithm for bacteraemia in patients with acute febrile illness. QJM. 2005;98:813-20.

36. Tokuda Y, Miyasato H, Stein GH, Kishaba T. The degree of chills for risk of bacteremia in acute febrile illness. Am J Med. 2005;118:1417. https://doi.org/ 10.1016/j.amjmed.2005.06.043.

37. Komatsu T, Takahashi E, Mishima K, et al. A simple algorithm for predicting bacteremia using food consumption and shaking chills: a prospective observational study. J Hosp Med. 2017;12:510-5.

38. Smith MD, Derrington P, Evans R, et al. Rapid diagnosis of bacteremic pneumococcal infections in adults by using the Binax NOW Streptococcus pneumoniae urinary antigen test: a prospective, controlled clinical evaluation. J Clin Microbiol. 2003;41:2810-3.

39. Boulware DR, Daley CL, Merrifield C, Hopewell PC, Janoff EN. Rapid diagnosis of pneumococcal pneumonia among HIV-infected adults with urine antigen detection. J Infect. 2007;55:300-9.

40. Horita N, Miyazawa N, Kojima R, et al. Sensitivity and specificity of the Streptococcus pneumoniae urinary antigen test for unconcentrated urine from adult patients with pneumonia: a meta-analysis. Respirology. 2013;18:1177-83.

41. Lee J, Song JU. Performance of pneumococcal urinary antigen test in patients with community-onset pneumonia: a propensity score-matching study. Korean J Intern Med. 2020;35:630-40. 